\title{
Skilled Nursing Facility Rehabilitation and Discharge to Home After Stroke
}

\author{
Walter P. Wodchis, PhD, Gary F. Teare, PhD, Gary Naglie, MD, Susan E. Bronskill, PhD, Sudeep S. Gill, MD, \\ Michael P. Hillmer, MSc, Geoff M. Anderson, MD, PhD, Paula A. Rochon, MD, Brant E. Fries, PhD
}

ABSTRACT. Wodchis WP, Teare GF, Naglie G, Bronskill SE, Gill SS, Hillmer MP, Anderson GM, Rochon PA, Fries BE. Skilled nursing facility rehabilitation and discharge to home after stroke. Arch Phys Med Rehabil 2005;86:442-8.

Objective: To determine the relation between rehabilitation therapy (RT) intensity and time to discharge home for stroke patients in skilled nursing facilities (SNFs).

Design: Retrospective cohort study. We used regression analyses, stratified by expected outcome, and propensity score adjustment.

Setting: All SNFs in Ohio, Michigan, and Ontario, Canada.

Participants: A cohort of residents, aged 65 and over, admitted from hospitals to SNFs with a diagnosis of stroke $(\mathrm{N}=23,824)$.

Interventions: Not applicable.

Main Outcome Measure: Time to discharge home from an SNF.

Results: RT was given to more than $95 \%$ of residents for whom discharge was expected within 90 days and to more than $60 \%$ of residents for whom discharge was uncertain or not expected. RT increased the likelihood of discharge to the community for all groups except those expected to be discharged within 30 days. The dose-response relation was strongest for residents with either an uncertain discharge prognosis or no discharge expected.

Conclusions: Postacute residents with an uncertain prognosis are an important target population for intensive RT.

Key Words: Nursing home; Patient discharge; Rehabilitation; Skilled nursing facilities.

(C) 2005 by American Congress of Rehabilitation Medicine and the American Academy of Physical Medicine and Rehabilitation

$\mathbf{R}$ EHABILITATION THERAPY (RT) for stroke patients discharged from hospitals is increasingly provided in skilled nursing facilities (SNFs). ${ }^{1}$ Stroke is the most common diagnosis for which older persons receive RT and studies indicate that more elderly stroke patients are discharged from hospitals to SNFs than to specialized rehabilitation hospitals. ${ }^{2,3}$ Kramer et $\mathrm{al}^{2}$ found that postacute stroke patients treated in

From the Toronto Rehabilitation Institute (Wodchis, Teare, Naglie); Institute for Clinical Evaluative Sciences (Wodchis, Teare, Bronskill, Gill, Anderson, Rochon); University of Toronto (Wodchis, Teare, Naglie, Bronskill, Hillmer, Anderson, Rochon); University Health Network (Naglie); Baycrest Centre for Geriatric Care (Gill, Hillmer, Rochon), Toronto, ON, Canada; University of Michigan (Fries); and Veterans Affairs, Ann Arbor, MI (Fries).

Supported by a Canadian Institutes of Health Research postdoctoral fellowship.

No commercial party having a direct interest in the results of the research supporting this article has or will confer a benefit on the author(s) or on any organization with which the author(s) is/are associated.

Reprint requests to Walter $\mathrm{P}$. Wodchis, $\mathrm{PhD}$, Toronto Rehabilitation Institute, Queen Elizabeth Ctr, 130 Dunn Ave, N236B, Toronto, ON M6K 2R7, Canada,

e-mail: wodchis.walter@torontorehab.on.ca.

0003-9993/05/8603-9078\$30.00/0

doi:10.1016/j.apmr.2004.06.067
SNFs are less likely to be discharged to the community than are those treated in higher intensity settings, and Kane et $\mathrm{al}^{3}$ found discharge and functional outcomes for those treated in SNFs to be worse than for those treated in both rehabilitation facilities and home-care settings. Identifying return to prior lifestyle in the community as the ultimate goal of rehabilitation, these studies raise serious questions about the effectiveness of poststroke rehabilitation care in SNFs. There is, in fact, very little evidence regarding the effect of rehabilitation on the timing of discharge to the community for poststroke SNF residents.

We studied the impact of rehabilitation delivered in SNFs, with a particular focus on whether higher intensities of RT increased the likelihood of a community discharge. Studies of stroke rehabilitation have suggested improved outcomes among patients treated in higher intensity settings (eg, specialized stroke units), ${ }^{2,4}$ but few have found strong evidence for a dose-response relation between therapy intensity and improved patient outcomes. ${ }^{5,6}$ Three studies of RT specific to nursing homes suggest that higher-intensity therapy may improve resident functioning ${ }^{7,8}$ and increase the likelihood of community discharge $^{9}$ for a general resident population. No studies have provided results for poststroke SNF residents. This gap in knowledge is the focus of the present study. This study describes the patterns of physical and occupational RT and examines the effect of RT intensity in SNFs on resident discharges to home.

\section{METHODS}

\section{Data}

Resident data for the study were obtained from an administrative database containing computerized nursing home Minimum Data Set v2.0 (MDS) resident assessments. The MDS is a comprehensive assessment containing more than 400 items related to resident diagnoses, functioning, and treatment. At a minimum, MDS assessments are collected within 2 weeks of admission, quarterly thereafter, and when there are significant changes in a resident's health status. The reliability and validity of the MDS for clinical practice and research purposes have been confirmed in repeated studies. ${ }^{10-14}$

Data from facilities in 3 adjacent regions of Canada and the United States expand the evaluation of treatment and outcomes and enhance the generality of this study's findings. Importantly, all residents in the study were assessed with the same assessment tool.

Data were extracted from the University of Michigan Assessment Archive Project, which contains data for all Michigan, Ohio, and Ontario residents, beginning with each region's implementation of electronic MDS v2.0 assessments (April 1, 1998 for Ohio; October 1, 1998 for Michigan; July 1, 1996 for Ontario). The MDS data derive from assessments that are mandated for all US SNFs and Ontario complex continuing care facilities (equivalent settings of care; hereafter all facilities are referred to as SNFs). MDS admission assessments must be made within the first 14 days of admission to an SNF. The 
assessment data are used to determine facility payment for residents and are subject to federal audit.

\section{Study Design and Patient Population}

We used a retrospective cohort design and examined time to discharge home based on treatment initiated on admission to the SNF. Although RT varies throughout the SNF stay, several studies have indicated the importance of early initiation of treatment. ${ }^{5}$ All residents were followed up from admission to discharge or the study's end date (December 31, 1999). We restricted the study population to residents aged 65 years and older who were admitted to SNFs directly from hospitals with a stroke diagnosis that affected care or outcomes (indicated on the MDS admission form; $\mathrm{N}=32,874$ ).

Five a priori exclusions were made. To select residents previously residing in the community, those assessed in the first 3 months of MDS assessment availability were excluded $(n=1566)$. Other exclusions were a terminal prognosis or cancer diagnosis (2531 excluded); hip fracture within the past 180 days (2420 excluded); US residents without Medicare Part A payment source (to control for potential differences in insurance benefits; 863 excluded); and missing data, more than 1000 minutes of weekly therapy (consistent with data audit procedures of the Centers for Medicare \& Medicaid Services), or inconsistent dates (eg, discharge before admission; 1670 excluded). Human subjects approval for this study was granted by the University of Michigan Institutional Review Board. The final sample included 23,824 residents.

\section{Outcome Measure}

The outcome of interest was time to discharge home (in days) for each resident admitted to an SNF. Standard MDS protocols require that the discharge date and the discharge destination be recorded for all residents. The dependent variable was defined as the SNF length of stay (LOS) at discharge to home. Observations were censored on the day that a resident was discharged to any other destination (acute hospital, rehabilitation hospital, other facility, death) or when they were lost to follow-up at the end of the study. Some patients had multiple admissions and discharges from the SNF, primarily because of hospital readmissions; in these cases, only the first admission and discharge were examined.

\section{Independent Variables}

The primary independent variable was the total weekly minutes of RT (the sum of physical and occupational therapy) provided to residents. The MDS assessment tracks the total amount of therapy in the 7 days before assessment. Federally mandated MDS assessments must be completed within 14 days of admission. To evaluate the potential for a nonlinear doseresponse relation between $\mathrm{RT}$ and discharge outcomes, RT minutes were categorized into 5 levels. The first level identified residents who received no therapy; 4 additional levels identified quartiles in the distribution of therapy minutes among all residents who received therapy.

\section{Risk Adjustment}

Confounding between therapy and other factors that can also affect discharge could bias the measured relation between therapy and discharge. ${ }^{15,16}$ We used a 3-part approach to control for potential confounding: multivariate risk-adjustment, stratified analyses, and propensity-score adjustment.

Multivariate adjustment. We included demographic and comorbid factors identified in prior rehabilitation studies and in stroke clinical guidelines to control for potential confounding between resident characteristics and the outcome..$^{2,4-7,17}$ Functional status scales were included to identify performance in activities of daily living (ADLs), using the MDS ADL hierarchy scale, ${ }^{13}$ and cognition, using the MDS Cognitive Performance Scale (CPS). ${ }^{18}$ Both the CPS and ADL scales are scored as independent (levels 0 or 1 on both scales), modified independence (levels 2, 3, or 4), and dependent (levels 5 or 6). We also measured the use of a cane and/or a wheelchair to move about. The MDS Depression Rating Scale ${ }^{19}$ was used to identify depressive symptoms. Mild depression is indicated by a rating of 3, 4, or 5 and major depression by a rating of 6 or higher.

We used 2 continuous scales to assess health instability and capacity for physical mobility training. MDS items indicating lack of voluntary movement in hands, arms, legs, and feet were summed to create a summary scale. Health instability was assessed using the MDS Changes in Health, End-Stage Disease, and Symptoms and Signs Scale, which was developed to predict mortality; it is similar to the Charlson index. ${ }^{20}$ Higher scores on both measures indicate greater impairment.

MDS care-planning protocols identify a "potential for functional rehabilitation" when residents (1) have any ADL impairment, or either the resident or staff believes the resident is capable of functional rehabilitation and (2) the resident can make decisions. ${ }^{21}$ Because this indicator was true for more than $90 \%$ of the sample, we restricted it by constraining the first part of the definition to those who required staff assistance in any ADL. The analysis also controlled for nurse-led rehabilitation activities, including range of motion (ROM) exercises; encouragement to improve independence in dressing, transferring, or eating; and training to return to the community.

Sample stratification. Although the extensive array of covariates addressed linear differences in patient characteristics that are thought to influence discharge, additional unobserved characteristics could potentially bias the estimated relation between RT and community discharge. For example, residents who are more likely to be discharged might be given more therapy. Thus, an observed positive relation between RT intensity and earlier discharge might have an endogenous rather than causal relation. We used a stratified analytical approach to control for this phenomenon. On the MDS admission assessment, clinical staff members assess whether they expect to discharge the resident within 30 days or between 30 and 90 days, whether the discharge prognosis is uncertain, or whether discharge is not expected (residents with expected deaths or hospitalizations are identified as "discharge not expected"). We conducted subsample analyses for each prognostic group.

Propensity adjustment. If resident characteristics included in the model correlate with treatment assignment (multicolinearity), estimates of the treatment effect will be biased by the amount of correlation between the treatment and correlated covariates. Propensity score adjustment is a powerful technique that can address systematic treatment selection based on observed characteristics. ${ }^{15,22}$ The propensity score is a measure of the relation between covariates and treatment assignment that is included as an independent variable in the regression of the outcome variable on the treatment. The propensity score isolates the correlation between covariates and treatment and allows an unbiased estimate of the treatment effect. We used this strategy to reduce the potential bias from confounding between observed resident characteristics and treatment. We used the full set of resident characteristics to estimate the propensity scores. For multilevel (dose-response) treatments, we estimated the multiple propensity score described by $\mathrm{Ru}$ bin $^{16}$ and Wang et al. ${ }^{23}$ The predicted probability identifying 


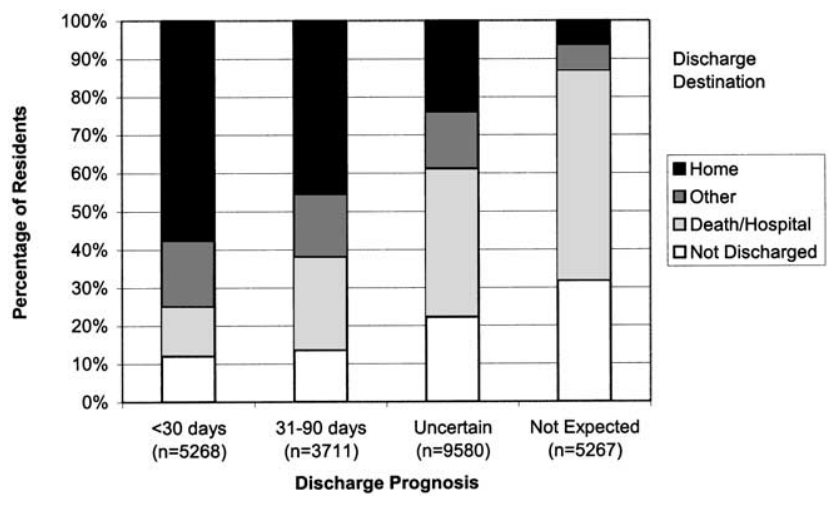

Fig 1. Resident discharges by discharge prognosis.

the level of therapy was categorized into quintiles and included as a covariate in the final model.

The propensity score was tested to examine whether covariates were balanced across treatment groups after adjusting for the propensity quintiles. The propensity scores were tested by statistically testing differences in resident characteristics between treatment groups in a model with only the propensity score and the characteristic. The ability of the propensity score to eliminate differences in treatment groups on each characteristic implies perfect balance.

\section{Analyses}

Cox proportional hazard regression models - one for each cohort of expected discharge-were used to examine the effect of RT on time of SNF stay until discharge to community. We estimated each hazard model using a full set of risk-adjustment covariates and then, similar to the strategy used by Kramer et al, ${ }^{2}$ used a backward stepwise elimination procedure to develop more parsimonious models while retaining significant predictors, thus ensuring that bias from correlation between covariates and our outcome (discharge home) was avoided. The backward elimination retained all variables with a $P$ value of .10 .

The relation between therapy and discharge was initially evaluated by comparing time to discharge home between residents with each level of therapy to those with no therapy. To test whether higher intensities of therapy were associated with a higher likelihood of discharge to the community, we also compared outcomes for only those residents who received some RT. For the latter analyses, residents receiving the lowest quartile of RT (1-175 weekly minutes) served as the reference group.

\section{RESULTS}

Figure 1 shows the discharge outcome for each stratum. A clear relation between discharge prognosis and discharge outcome is apparent. Discharges to home were most common for residents with an earlier expected discharge. Only $6 \%$ of residents with no discharge expected were discharged home.

The distribution of total weekly RT minutes is shown in figure 2, stratified by discharge prognosis. The largest group of residents had an uncertain discharge prognosis $(n=9580)$, followed by nearly equal numbers with discharge not expected $(n=5267)$ and discharge within 30 days $(n=5268)$; 3711 residents had an expected discharge between 31 and 90 days. Most residents received some RT, and higher RT levels were more common in residents with an earlier discharge prognosis. However, the distribution of RT among residents who received some therapy was relatively homogenous, with surprisingly high representation of intensive therapy among those with an uncertain prognosis or no discharge expected.

Table 1 identifies the characteristics of residents with each discharge prognosis stratum. Several characteristics indicated a strong association with prognostic stratum. Desiring discharge and having a discharge support person were both highly related to discharge prognosis. Seizures, aphasia, dementia, pressure ulcers, and use of feeding tubes were more prevalent among residents with a less positive discharge prognosis. Functional status scales uniformly indicate that patients given an early discharge prognosis were less severely impaired. Nurse-led rehabilitation in training for community residence was 6 times more prevalent for residents with an early discharge prognosis compared with those with no discharge expected. Other characteristics did not appear to have a systematic relation with discharge prognosis

\section{Multivariate Results}

Multivariate analyses were conducted first to identify the overall effect of RT on the likelihood of discharge to the community and then to test for the presence of a doseresponse relation between RT and time to discharge home. In the analysis for all residents (table 2), the following diagnoses, if present on admission to the SNF, did not affect discharge in any regression model (and were deleted by the stepwise procedures): congestive heart failure, hypertension, peripheral vascular disease, seizures, urinary tract infection, and thrombosis. In addition, the level of assistance required for dressing, eating, and transferring (on admission to the SNF) also did not affect the discharge destination, presumably because these are the deficits for which rehabilitation was given. (Full results are available from the authors on request.)

Table 2 presents the results of a Cox proportional hazard regression on time to discharge home for all residents, stratified by discharge prognosis. Within each discharge prognosis, residents who received no RT on admission to the SNF served as the reference group. Estimates control for treatment propensity and comorbid conditions. Propensity score adjustment was effective in controlling for most systematic differences between treatment groups within each prognostic stratum. The comparisons where propensity

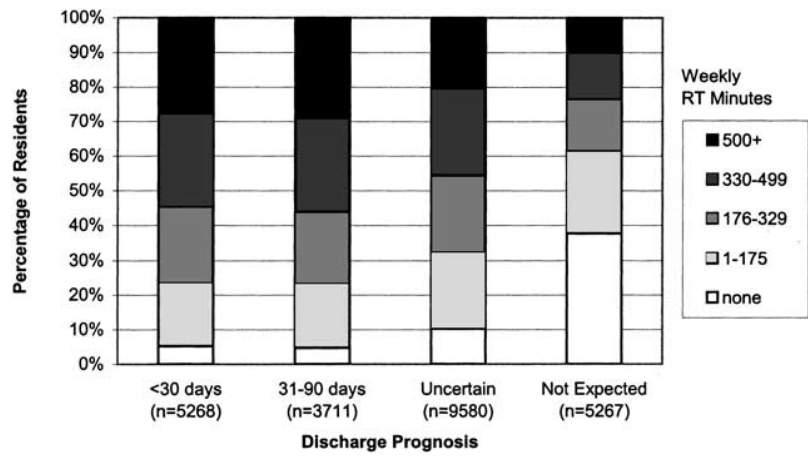

Fig 2. Distribution of therapy minutes by discharge prognosis. 
Table 1: Resident Characteristics by Discharge Prognosis

\begin{tabular}{|c|c|c|c|c|}
\hline \multirow[b]{3}{*}{ Characteristics } & \multicolumn{4}{|c|}{ Discharge Prognosis } \\
\hline & $\begin{array}{l}<30 \text { Days } \\
(n=5268)\end{array}$ & $\begin{array}{c}\text { 31-90 Days } \\
(\mathrm{n}=3711)\end{array}$ & $\begin{array}{l}\text { Uncertain } \\
(n=9580)\end{array}$ & $\begin{array}{l}\text { Not Expected } \\
\quad(n=5267)\end{array}$ \\
\hline & \multicolumn{4}{|c|}{ Column Prevalence } \\
\hline \multicolumn{5}{|l|}{ Demographics } \\
\hline \multicolumn{5}{|l|}{ Age (y) } \\
\hline $65-74$ & 26 & 24 & 23 & 20 \\
\hline $75-84$ & 47 & 48 & 48 & 45 \\
\hline $85+$ & 27 & 28 & 30 & 35 \\
\hline Lived alone prior & 35 & 36 & 33 & 24 \\
\hline Male & 39 & 40 & 40 & 39 \\
\hline Married & 38 & 38 & 37 & 32 \\
\hline Discharge support person & 95 & 92 & 68 & 5 \\
\hline Resident desires discharge & 93 & 92 & 68 & 12 \\
\hline Ohio & 48 & 21 & 23 & 32 \\
\hline Michigan & 40 & 53 & 58 & 49 \\
\hline Ontario & 12 & 26 & 19 & 19 \\
\hline \multicolumn{5}{|l|}{ Comorbidity } \\
\hline Deep vein thrombosis & 2 & 2 & 2 & 2 \\
\hline Seizure disorder & 8 & 6 & 8 & 10 \\
\hline Hemiplegia/hemiparesis & 27 & 37 & 36 & 33 \\
\hline Aphasia & 8 & 10 & 14 & 18 \\
\hline Parkinson's disease & 4 & 4 & 5 & 5 \\
\hline Hypertension & 65 & 63 & 64 & 60 \\
\hline ASHD & 22 & 20 & 21 & 24 \\
\hline $\mathrm{CHF}$ & 27 & 24 & 29 & 33 \\
\hline PVD & 11 & 11 & 11 & 14 \\
\hline Dementia & 16 & 18 & 29 & 42 \\
\hline Pneumonia & 10 & 9 & 12 & 14 \\
\hline Urinary tract infection & 19 & 21 & 23 & 23 \\
\hline Monitor acute condition & 51 & 47 & 52 & 48 \\
\hline Pressure ulcers & 29 & 37 & 47 & 63 \\
\hline \multicolumn{5}{|l|}{ Scales } \\
\hline \multicolumn{5}{|l|}{$A D L$} \\
\hline Independent & 12 & 7 & 4 & 3 \\
\hline Modified & 56 & 56 & 44 & 32 \\
\hline Dependent & 32 & 38 & 52 & 65 \\
\hline \multicolumn{5}{|l|}{ CPS } \\
\hline Independent & 53 & 47 & 26 & 15 \\
\hline Modified & 41 & 46 & 58 & 51 \\
\hline Dependent & 6 & 8 & 16 & 34 \\
\hline \multicolumn{5}{|l|}{ Depression } \\
\hline None & 92 & 89 & 86 & 87 \\
\hline Mild & 7 & 8 & 11 & 10 \\
\hline Major & 2 & 2 & 3 & 3 \\
\hline Voluntary mobility (range, $0-4$ ) & $1.36 \pm 1.8$ & $1.82 \pm 1.9$ & $2.06 \pm 2.2$ & $2.43 \pm 2.4$ \\
\hline Health instability (range, 0-6) & $1.97 \pm 1.1$ & $1.95 \pm 1.1$ & $2.09 \pm 1.2$ & $1.79 \pm 1.3$ \\
\hline Use of cane & 67 & 53 & 37 & 23 \\
\hline Use of wheelchair & 45 & 70 & 70 & 64 \\
\hline Rehabilitation potential & 37 & 43 & 32 & 18 \\
\hline Feeding tube & 7 & 9 & 18 & 28 \\
\hline Speech therapy & 31 & 41 & 44 & 31 \\
\hline \multicolumn{5}{|l|}{ Nurse-led care } \\
\hline Training for community & 56 & 54 & 33 & 10 \\
\hline Active ROM & 9 & 15 & 11 & 12 \\
\hline Passive ROM & 6 & 13 & 14 & 19 \\
\hline Dressing/grooming training & 11 & 19 & 13 & 10 \\
\hline Eating/swallowing training & 4 & 8 & 8 & 7 \\
\hline Transfer training & 12 & 22 & 14 & 10 \\
\hline
\end{tabular}

NOTE. Values are percent or mean \pm standard deviation.

Abbreviations: ASHD, arteriosclerotic heart disease; CHF, congestive heart failure; PVD, peripheral vascular disease. 
Table 2: Multivariate Relative Risk for Discharge to Home

\begin{tabular}{|c|c|c|c|c|c|}
\hline \multirow[b]{2}{*}{ Weekly RT Minutes } & \multicolumn{4}{|c|}{ Discharge Prognosis on Admission } & \multirow[b]{2}{*}{ All Residents } \\
\hline & $<30$ Days & 31-90 Days & Uncertain & Not Expected & \\
\hline None (reference) & 1.00 & 1.00 & 1.00 & 1.00 & 1.00 \\
\hline $1-175$ & $1.06(0.84-1.34)$ & $1.54^{\dagger}(1.08-2.20)$ & $1.19(0.94-1.51)$ & $1.29(0.86-1.92)$ & $1.20^{\dagger}(1.04-1.38)$ \\
\hline $176-329$ & $0.98(0.77-1.23)$ & $1.32(0.92-1.89)$ & $1.46^{\dagger}(1.14-1.86)$ & $1.51 *(0.96-2.35)$ & $1.37^{\ddagger}(1.18-1.58)$ \\
\hline 330-499 & $1.09(0.86-1.37)$ & $1.56^{\dagger}(1.09-2.26)$ & $1.57^{\ddagger}(1.23-2.02)$ & $2.18^{\ddagger}(1.36-3.49)$ & $1.64^{\ddagger}(1.42-1.90)$ \\
\hline $500+$ & $1.07(0.84-1.36)$ & $1.78^{\ddagger}(1.23-2.57)$ & $1.67^{\ddagger}(1.30-2.16)$ & $2.23^{\ddagger}(1.38-3.61)$ & $1.58^{\ddagger}(1.36-1.83)$ \\
\hline
\end{tabular}

NOTE. Values are relative risk (95\% confidence interval [CI]).

${ }^{*} P<.10 ;{ }^{\dagger} P<.05 ;{ }^{\ddagger} P<.01$ (significance of difference from reference category).

matching may not have provided sufficient matching are comparisons of the fourth quartile of therapy for residents who received no therapy among residents with an expected discharge within 30 days or between 31 and 90 days. We accounted for the fact that the propensity score alone might not provide complete control for resident differences across all treatment comparisons by including additional resident characteristics, selected by the backward elimination procedure, along with the propensity score in the multivariate analyses.

RT increased the likelihood of discharge to community except for residents with an expected discharge within 30 days, for whom it had no significant effect on time to discharge home. For residents whose discharge was expected between 31 and 90 days, significant results indicated that RT increased the likelihood of discharge home by between $54 \%$ and $78 \%$, although the second quartile of RT was not significant. For residents with an uncertain prognosis, all but the lowest levels of RT had a significant and positive effect on the likelihood of discharge home. For residents with no discharge expected, RT in the second, third, and fourth quartiles also had a positive and significant effect on time to discharge home. The likelihood of discharge home was more than 2 times higher for such residents who received 330 or more minutes of RT each week, compared with those who received none. Full sample results are weighted by the largest group (uncertain prognosis). The full set of results, taken together, suggests a larger effect for higher RT intensity, particularly for residents with relatively negative initial prognoses.

\section{Dose-Response Results}

Further analyses were conducted to provide a direct test of a dose-response effect of RT on time to discharge home. This analysis excluded residents with no RT and compared outcomes for residents with higher levels of RT with outcomes for residents receiving the lowest quartile of RT. The results are shown in table 3 .
Propensity adjustment was particularly effective for the dose-response analyses. Fifteen of 24 estimated propensity scores provided perfectly balanced characteristics, and the remaining 9 had only 1 characteristic that remained significantly different (primarily Ontario residence, where ultrahigh rehabilitation is relatively rare). As above, additional multivariate characteristics were included alongside the propensity quintiles in estimating the dose-response effects.

No significant dose response was found for residents either with a discharge expected within 30 days or between 31 and 90 days. For residents with an uncertain prognosis, increased RT intensity was positively and significantly associated with an increased likelihood of discharge home. A positive dose-response pattern was also observed in the discharge-not-expected group, although only the highest quartile attained a $P$ value of less than .05 . The results suggest but do not universally support a dose-response relationship.

\section{Sensitivity Analyses}

To ensure that our results were not sensitive to events that occurred late in the SNF stay, we did additional analyses to examine only discharges within the first 180 days and censored all residents not discharged by this time. Risk ratios were within .05 of all the significant estimates presented in tables 2 and 3. Multivariate analyses were also repeated separately for each region of residence. Estimated risk ratios were within the confidence limits of estimates presented in tables 2 and 3, although reduced power (fewer events) meant that significance was not replicated for all the results that were significant in the combined population. The following differences were observed: Ohio-only results were not significant in the 31-to-90-days discharge stratum, and Ontario results were not significant in the no-discharge-expected stratum and were only significant for the highest RT level in the uncertain-discharge stratum.

Table 3: Dose-Response Relative Risk for Discharge to Home

\begin{tabular}{|c|c|c|c|c|c|}
\hline \multirow[b]{2}{*}{ Weekly RT Minutes } & \multicolumn{4}{|c|}{ Discharge Prognosis on Admission } & \multirow[b]{2}{*}{ All Residents } \\
\hline & $<30$ Days & 31-90 Days & Uncertain & Not Expected & \\
\hline 1-175 (reference) & 1.00 & 1.00 & 1.00 & 1.00 & 1.00 \\
\hline $176-329$ & $1.01(0.89-1.14)$ & $1.08(0.91-1.28)$ & $1.15^{\dagger}(1.00-1.32)$ & $1.03(0.72-1.46)$ & $1.09^{\dagger}(1.00-1.17)$ \\
\hline 330-499 & $0.99(0.87-1.13)$ & $1.13(0.95-1.34)$ & $1.16^{\dagger}(1.01-1.34)$ & $1.42 *(0.98-2.06)$ & $1.14^{\ddagger}(1.06-1.24)$ \\
\hline $500+$ & $1.01(0.89-1.16)$ & $1.12(0.94-1.33)$ & $1.28^{\ddagger}(1.11-1.49)$ & $1.52^{\dagger}(1.05-2.21)$ & $1.16^{\ddagger}(1.07-1.26)$ \\
\hline
\end{tabular}

NOTE. Values are relative risk $(95 \% \mathrm{Cl})$.

${ }^{*} P<.10 ;{ }^{\dagger} P<.05 ;{ }^{\ddagger} P<.01$ (significance of difference from reference category). 


\section{DISCUSSION}

Prior meta-analyses and clinical trials have provided mixed evidence of the relation between therapy intensity and LOS. Most studies have examined differences between specialized units and conventional multidisciplinary care, with an inherent presumption that specialized care provides a higher intensity of therapy. ${ }^{4}$ Although meta-analyses have found statistically significant effect of higher-intensity therapy, the overall benefits appear to be quite small. ${ }^{5,6,24}$ Individual trials have found stronger effects, but only for shortterm outcomes; long-term outcomes appear to be insensitive to treatment intensity. ${ }^{4}$

Our study provides new insight into the relation between RT intensity and discharge outcomes in SNFs. In particular, we found that there may be little effect of very high RT intensity on time to discharge home when the short-term prognosis is positive. Residents with less optimistic prognoses, on the other hand, may benefit substantially from additional RT. This highlights the importance of targeting patients who might most benefit from higher levels of RT. New analytic strategies such as clustering techniques are increasing the potential to identify such residents. ${ }^{25}$ Improving evidence-based targeting criteria is an important next step.

The lack of an observed relation between RT and time to discharge home among residents with the earliest expected discharge may be attributable to several possible causes: (1) there are very few residents who do not receive RT in this population; (2) there is little variation in the time to discharge to the community among residents in this population; (3) these residents may already be ready for, or have sufficient existing support for, transition to the community and are merely in the SNF to stabilize medical complications or to wait for necessary community arrangements to be made (eg, home modifications); or (4) higher levels of RT may provide no additional benefit to these residents with respect to the timing of discharge. Intensive therapy in the earliest expected-discharge subgroups may, however, affect outcomes not measured in this study, such as instrumental ADLs or other skills that enhance performance in the home but are not required for home discharge. Additional research is also needed to evaluate the effect of RT intensity on long-term functional outcomes.

Although point-estimates in the dose-response analysis were largest in the no-discharge-expected group, $P$ values were higher than the common standard of .05. An important reason is lack of power in the sample; the latter group had only 286 residents who received therapy and were discharged to home.

As an observational study using administrative data sources, there are limitations to the causal inferences that can be drawn from our study. Although several associations (and nonassociations) were found, the causal relation between therapy and discharge outcomes cannot be confirmed. In the statistical analyses we were careful to identify and control for confounding factors; however, only observable characteristics can be adjusted for, and unobserved differences in populations may confound the results reported here. No statistical method can address hidden biases due to unobserved or unrecorded differences between residents who receive different RT intensities. A carefully constructed observational study nonetheless can inform debate and provide information about population groups that might be excluded or rare in randomized controlled trials. Such trials are required to examine more thoroughly the relations between therapy intensity and resident outcomes. Although stroke is the most common reason for RT, further research should also examine other common conditions, such as hip fractures.

\section{CONCLUSIONS}

The association between the intensity of therapy and resident discharges to community settings found in this study provides new information about the effectiveness of therapy for postacute stroke patients treated in SNFs. Based on our results, greater attention should be given to residents with an uncertain or negative discharge prognosis. We suggest that the marginal benefit to these residents may be greater than the marginal benefit experienced by residents with a discharge expected within 90 days.

\section{References}

1. Murray PK, Singer ME, Fortinsky R, Russo L, Cebul RD. Rapid growth of rehabilitation services in traditional community-based nursing homes. Arch Phys Med Rehabil 1999;80:372-8.

2. Kramer AM, Steiner JF, Schlenker RE, et al. Outcomes and costs after hip fracture and stroke. A comparison of rehabilitation settings. JAMA 1997;277:396-404.

3. Kane RL, Chen Q, Finch M, Blewett L, Burns R, Moskowitz M. The optimal outcomes of post-hospital care under Medicare. Health Serv Res 2000;35:615-61.

4. Teasell R, Doherty T, Speechley M, Foley N, Bhogal SK. Evidence-based review of stroke rehabilitation. London $(\mathrm{ON})$ : St. Joseph's Health Care; 2002.

5. Cifu DX, Stewart DG. Factors affecting functional outcome after stroke: a critical review of rehabilitation interventions. Arch Phys Med Rehabil 1999;80(5 Suppl 1):S35-9.

6. Kwakkel G, Wagenaar RC, Koelman TW, Lankhorst GJ, Koetsier JC. Effects of intensity of rehabilitation after stroke. A research synthesis. Stroke 1997;28:1550-6.

7. Przybylski BR, Dumont ED, Watkins ME, Warren SA, Beaulne AP, Lier DA. Outcomes of enhanced physical and occupational therapy service in a nursing home setting. Arch Phys Med Rehabil 1996;77:554-61.

8. Chiodo LK, Gerety MB, Mulrow CD, Rhodes MC, Tuley MR The impact of physical therapy on nursing home patient outcomes. Phys Ther 1992;72:168-75.

9. Arling G, Williams AR, Kopp D. Therapy use and discharge outcomes for elderly nursing home residents. Gerontologist 2000; 40:587-95

10. Morris JN, Nonemaker S, Murphy K, et al. A commitment to change: revision of HCFA's RAI. J Am Geriatr Soc 1997;45: 1011-6.

11. Morris JN, Fries BE, Morris SA. Scaling ADLs within the MDS J Gerontol A Biol Sci Med Sci 1999;54:M546-53.

12. Sgadari A, Morris JN, Fries BE, et al. Efforts to establish the reliability of the Resident Assessment Instrument. Age Ageing 1997;26(Suppl 2):27-30.

13. Frederiksen K, Tariot P, De Jonghe E. Minimum Data Set Plus (MDS+) scores compared with scores from five rating scales. J Am Geriatr Soc 1996;44:305-9.

14. Hawes C, Morris JN, Phillips CD, Mor V, Fries BE, Nonemaker S. Reliability estimates for the Minimum Data Set for nursing home resident assessment and care screening (MDS). Gerontologist 1995;35:172-8.

15. Rosenbaum PR, Rubin DB. The central role of the propensity score in observational studies for causal effects. Biometrika 1983; 70:41-55.

16. Rubin DB. Estimating causal effects from large data sets using propensity scores. Ann Intern Med 1997;127:757-63.

17. Joseph CL, Wanlass W. Rehabilitation in the nursing home. Clin Geriatr Med 1993;9:859-71. 
18. Morris JN, Fries BE, Mehr DR, et al. MDS Cognitive Performance Scale. J Gerontol 1994;49:M174-82.

19. Burrows AB, Morris JN, Simon SE, Hirdes JP, Phillips C. Development of a minimum data set-based depression rating scale for use in nursing homes. Age Ageing 2000;29:165-72.

20. Hirdes JP, Frijters DH, Teare GF. The MDS-CHESS scale: a new measure to predict mortality in institutionalized older people. J Am Geriatr Soc 2003;51:96-100.

21. Centers for Medicare and Medicaid Services. Revised long term care facility resident assessment instrument user's manual. Baltimore: CMS; 2002.
22. D'Agostino RB Jr. Propensity score methods for bias reduction in the comparison of a treatment to a non-randomized control group. Stat Med 1998;17:2265-81.

23. Wang J, Donnan PT, Steinke D, MacDonald TM. The multiple propensity score for analysis of dose-response relationships in drug safety studies. Pharmacoepidemiol Drug Saf 2001;10:105-11.

24. Langhorne P, Wagenaar RC, Partridge C. Physiotherapy after stroke: more is better? Physiother Res Int 1996;1:75-88.

25. Eastwood EA, Magaziner J, Wang J, et al. Patients with hip fracture: subgroups and their outcomes. J Am Geriatr Soc 2002;50: 1240-9. 